OPEN ACCESS

Edited by:

Zsuzsa Kaldy

University of Massachusetts Boston

USA

Reviewed by:

Bradley R. Postle,

University of Wisconsin-Madison,

USA

Thomas B. Christophel, Charité - Universitätsmedizin Berlin,

Germany

Hoi Chung Leung,

Stony Brook University, USA

*Correspondence:

Chris I. Baker

bakerchris@mail.nih.gov

Received: 17 September 2015 Accepted: 08 January 2016 Published: 15 February 2016

Citation:

Lee S-H and Baker Cl (2016)

Multi-Voxel Decoding and the Topography of Maintained Information During Visual Working Memory.

Front. Syst. Neurosci. 10:2. doi: 10.3389/fnsys.2016.00002

\section{Multi-Voxel Decoding and the Topography of Maintained Information During Visual Working Memory}

\author{
Sue-Hyun Lee ${ }^{1,2}$ and Chris I. Baker ${ }^{2 *}$ \\ ${ }^{1}$ Department of Bio and Brain Engineering, College of Engineering, Korea Advanced Institute of Science and Technology \\ (KAIST), Daejeon, South Korea, ${ }^{2}$ Laboratory of Brain and Cognition, National Institute of Mental Health, National Institutes of \\ Health, Bethesda, MD, USA
}

The ability to maintain representations in the absence of external sensory stimulation, such as in working memory, is critical for guiding human behavior. Human functional brain imaging studies suggest that visual working memory can recruit a network of brain regions from visual to parietal to prefrontal cortex. In this review, we focus on the maintenance of representations during visual working memory and discuss factors determining the topography of those representations. In particular, we review recent studies employing multi-voxel pattern analysis (MVPA) that demonstrate decoding of the maintained content in visual cortex, providing support for a "sensory recruitment" model of visual working memory. However, there is some evidence that maintained content can also be decoded in areas outside of visual cortex, including parietal and frontal cortex. We suggest that the ability to maintain representations during working memory is a general property of cortex, not restricted to specific areas, and argue that it is important to consider the nature of the information that must be maintained. Such information-content is critically determined by the task and the recruitment of specific regions during visual working memory will be both task- and stimulus-dependent. Thus, the common finding of maintained information in visual, but not parietal or prefrontal, cortex may be more of a reflection of the need to maintain specific types of visual information and not of a privileged role of visual cortex in maintenance.

\section{Keywords: working memory, short term memory, multivoxel pattern analysis (MVPA), visual imagery, visual working memory, fMRI}

\section{INTRODUCTION}

Working memory commonly refers to our ability to maintain and manipulate stimulus representations, typically for a short period of time, in the absence of the ongoing presence of that stimulus (Baddeley and Hitch, 1974). For example, holding a phone number in mind prior to pressing the buttons on the phone. In vision, working memory can involve diverse types of maintained content from complex forms such as faces and objects to fine visual details such as specific orientations or colors. The neural basis of visual working memory has long been the subject of debate and while multiple brain areas, from visual cortex, including primary visual cortex (V1) and the middle temporal area (MT), to the parietal, temporal and prefrontal 
cortices have been implicated in visual working memory (Wager and Smith, 2003), the functional roles these regions play has been controversial. Typically, theories have distinguished different processes that might be involved in visual working memory (Eriksson et al., 2015), making a distinction between stimulus representation or storage and executive or top down control, and have tried to map those distinctions onto specific brain regions. Various accounts posit that there is a working memory system separate from other memory or perception systems (e.g., Baddeley, 2012), that prefrontal cortex is involved in both maintenance and executive control (e.g., Funahashi et al., 1989, 1993; Chafee and Goldman-Rakic, 1998; Constantinidis et al., 2001), or that information is maintained in posterior cortex with prefrontal cortex primarily involved in top-down control of those regions (for recent review, see D'Esposito and Postle, 2015). In this review, we will focus on recent evidence from human functional magnetic resonance imaging (fMRI) studies identifying the substrates of maintained representations during visual working memory.

The terms "visual working memory" and "visual shortterm memory" are often used interchangeably. One of the key components of working memory is indeed the short-term maintenance of visual representations. However, working memory is often used to describe not just maintenance of representations, but internal manipulation of those representations as well (for recent discussion, see Marois, 2015; Postle, 2015a). In this review, we will refer to "visual working memory", following many of the studies that we cite, although our primary focus is on the maintenance of visual representations. Such maintenance can occur in many different contexts. For example, a participant might be asked to remember a stimulus that is briefly flashed on the screen (e.g., Serences et al., 2009). Alternatively, a participant might be cued to recall a recently presented stimulus, out of two or more alternatives, and then asked to remember that stimulus over a delay period (e.g., Harrison and Tong, 2009). However, the representations that are being maintained need not be accessed from recent sensory experience, but can also be retrieved from long-term memory, allowing further manipulation of the remembered content in such a way that makes it useful for ongoing behavior. In this light, visual working memory may share mechanisms with visual imagery (Albers et al., 2013; Tong, 2013) and even the accessing of conceptual knowledge (Martin, 2007, 2015).

In this review, we will highlight that to understand the engagement of particular regions during working memory, it is important to consider the nature of the stimulus representations that are being maintained. We will use the term "information" to refer to the specific aspects of the presented stimulus that are relevant to task performance and must therefore be remembered over the delay period. Thus, "information" does not necessarily refer to the entire stimulus itself or even to sensory properties of the stimulus. The maintained information could be one aspect of a visually presented stimulus (e.g., color, but not orientation, of a grating stimulus), or an abstraction from the stimulus (e.g., category). Further, the same information could be contained in very different underlying representations. For example, stimulus position could be maintained either in a visual representation (e.g., in V1) or a motor representation for an upcoming eye movement.

The fMRI studies we focus on have employed multivoxel pattern analysis (MVPA) techniques to decode maintained representations during the delay periods of working memory tasks. By "decoding" we simply mean that the BOLD response measured with fMRI has been used to infer the information that is represented. Many of these studies have revealed maintained representations in visual cortex (e.g., V1-V4, MT), supporting a role of sensory, not prefrontal, cortex in maintenance. However, there is some evidence for maintenance outside of visual cortex (including posterior parietal and prefrontal cortex) and here, we suggest that the ability to maintain information is a general property of cortex, not limited to specific regions. We argue that the predominance of studies revealing maintained representations in early visual cortex reflects the stimuli and task that have been probed. Specifically, the recruitment of any region will reflect the particular information that must be maintained as determined by the task context and the behavioral goals. Thus, working memory is best understood as a highly distributed process wherein information can be maintained in any systems engaged in the initial perceptual processing. This includes not just sensory cortex, but any region contributing to the initial percept, including parietal and frontal areas.

\section{DECODING MAINTAINED REPRESENTATIONS}

The notion that information is maintained in sensory regions during visual working memory has been referred to as the "sensory recruitment" hypothesis (Pasternak and Greenlee, 2005). Early support for this view came from perceptual discrimination studies in which participants had to detect whether a sample stimulus (varying in spatial frequency, orientation, or motion stimulus) matched a test stimulus presented after a brief delay (Dupont et al., 1998; Magnussen and Greenlee, 1999). Irrelevant stimuli presented during the delay were found to interfere with discrimination performance in a feature-selective manner, suggesting that the mechanisms involved in maintaining the representation of the sample stimulus are linked to those involved in perceptual processing (Magnussen et al., 1991; Magnussen and Greenlee, 1992).

However, physiology (e.g., Funahashi et al., 1989, 1993; Miller et al., 1996; Constantinidis et al., 2001) and early fMRI (e.g., Zarahn et al., 1997; Courtney et al., 1998; Jha and McCarthy, 2000; Leung et al., 2002) studies shifted the emphasis away from sensory cortex to prefrontal cortex with the observation of elevated activity during the delay period that spanned intervening stimuli. While it was appealing to equate maintained activity with maintained representations, the mere presence of elevated activity does not indicate the nature of the underlying processing (Curtis and D'Esposito, 2003; Sreenivasan et al., 2014a). Further, such increased activity can also be found in posterior brain areas (Ranganath and D'Esposito, 2005) for both simple (Greenlee et al., 2000) and complex (Courtney et al., 1997; 
Druzgal and D'Esposito, 2003; Ranganath et al., 2004; Oh and Leung, 2010) visual features.

An alternative approach, focusing on the capacity limit of working memory, highlighted the potential role of parietal cortex. In particular, regions in parietal cortex exhibit activity which tracks the number of items held in memory and correlates with apparent capacity limitations (Linden et al., 2003; Todd and Marois, 2004, 2005; Vogel and Machizawa, 2004; Xu and Chun, 2006; Harrison et al., 2010). Further, Mitchell and Cusack (2008) found correlation with capacity-based regressors not only in parietal cortex but also in some prefrontal areas. While these findings suggest a link between parietal (and possibly prefrontal) cortex and working memory capacity, they do not indicate that the representations are maintained in these regions.

Recent fMRI studies have now provided more compelling evidence for the sensory recruitment model by focusing on whether the responses in a given region are specific to the maintained information (D'Esposito and Postle, 2015). Such studies have taken advantage of the development of MVPA techniques (for reviews, see Norman et al., 2006; Serences and Saproo, 2012; Haynes, 2015), which focus on the patterns of response across voxels rather than the average magnitude (see Table 1 for a summary of studies). In these studies, the BOLD responses in a given region are used to infer or "decode" the nature of the underlying representation. For example, Harrison and Tong (2009) presented participants with two serially presented gratings, followed by a retro-cue ("1" or a "2") indicating whether they had to remember the first or second grating. A test grating was presented after a further delay of $11 \mathrm{~s}$ and participants had to indicate whether it was rotated clockwise or anticlockwise relative to the cued grating. There were three key findings. First, during the delay period, the patterns of BOLD response in early visual cortex (V1-V4) could be used to decode the orientation of the grating held in memory, suggesting that early visual cortex holds a specific representation of the maintained orientation. Second, this decoding was possible even when there was no elevated activity during the delay period, suggesting that elevated activity is not necessary for the maintenance of orientation information. Third, the patterns of response observed during the delay period were similar to those evoked by physically presented gratings, suggesting that the maintained representations are strongly related to perceptual representations in these areas.

Support for the maintenance of representations in early visual cortex has also been provided by an alternative approach in which the response properties of individual voxels are explicitly modeled. For example, Ester et al. (2013) fit a model (often termed an encoding model) of orientation selectivity, based on a set of eight orientation-selective response functions "channels", to each voxel in early visual areas (following the approach of Brouwer and Heeger, 2009, 2011). Then, based on the response pattern across voxels (in independent data), they could reconstruct images reflecting the information content in a given area during the delay period of the task. This analysis revealed graded response profiles in V1 and V2 that peaked for the remembered orientation and was only present when explicit memory was required.

The ability to decode maintained orientation information in early visual cortex during visual working memory has now been replicated multiple times, supporting the three key findings described above (Ester et al., 2009, 2015; Serences et al., 2009; Sneve et al., 2012; Albers et al., 2013; Pratte and Tong, 2014). Further, the precision of the orientation representations in early visual cortex, measured as memory load is varied, reflects behavioral performance (Ester et al., 2013; see also Emrich et al., 2013). Beyond orientation, decoding of maintained representations has also been reported in early visual cortex for contrast (Xing et al., 2013), location (Sprague et al., 2014), motion (Riggall and Postle, 2012; Emrich et al., 2013), color (Serences et al., 2009), and color patterns (Christophel et al., 2012, 2015).

In all of these cases, the information that can be decoded during visual working memory is the kind of information (e.g., orientation, color, contrast) that is well represented by the underlying stimulus feature-selectivity in early visual cortex. Similarly, other areas of visual cortex with more specialized feature-selectivity during perception have demonstrated maintenance of information corresponding to that selectivity. For example, decoding of simple (Riggall and Postle, 2012; Emrich et al., 2013) and complex motion information (Christophel and Haynes, 2014) has been reported in the human MT complex (MT+) that is highly selective for stimulus motion. Further, in studies that have tested working memory for complex images such as objects, scenes and faces, decoding of maintained information has been reported in category-selective occipitotemporal cortex (Linden et al., 2012; Han et al., 2013; Lee et al., 2013; Nelissen et al., 2013; Sreenivasan et al., 2014b). However, it is important to note that in many of these cases while the task required within-category information (e.g., individual faces or scenes), decoding was at the level of category (e.g., faces vs. scenes, see Table 1). Thus, the ability to maintain representations appears to be a general property of visual cortex, with regions maintaining representations of those stimuli that match their underlying stimulus-selectivity.

It is important to realize, however, that the maintenance of content during delay periods is not simply a passive reflection of stimulus properties. The nature of the information maintained is critically dependent on the task, which determines the specific information that is required for successful performance. For example, Serences et al. (2009) presented colored oriented gratings and varied whether color or orientation was relevant for the discrimination to be made after the delay. They found that both orientation and color could be decoded from V1 during the delay, but only when that specific feature information was taskrelevant. Similarly, while there is some evidence that orientation information is maintained throughout V1, not just in the part of the retinotopic map corresponding to the stimulus location in the visual field (Ester et al., 2009, 2015), location-specific orientation information can be decoded when both location and orientation are task-relevant (Pratte and Tong, 2014). Consistent with this, Lee et al. (2013) reported decoding of object identity in high-level visual cortex only when the visual properties of the presented stimuli were task-relevant. 
TABLE 1 | Summary of studies demonstrating multi-voxel decoding of information during visual working memory.

\begin{tabular}{|c|c|c|c|c|}
\hline Reference & Stimuli & $\begin{array}{l}\text { Task-relevant } \\
\text { information }\end{array}$ & $\begin{array}{l}\text { Information } \\
\text { decoded }\end{array}$ & $\begin{array}{l}\text { Cortical regions } \\
\text { allowing decoding }\end{array}$ \\
\hline
\end{tabular}

Ester et al. (2009)

Harrison and Tong (2009)

Serences et al. (2009)

Christophel et al. (2012)

Jerde et al. (2012)

Linden et al. (2012)

Riggall and Postle (2012)

Sneve et al. (2012)

Albers et al. (2013)

Emrich et al. (2013)
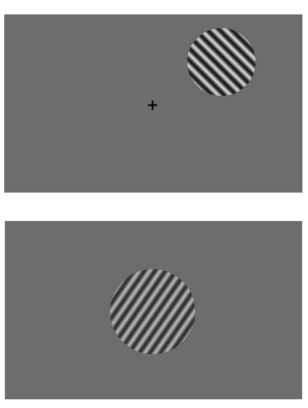

Orientation

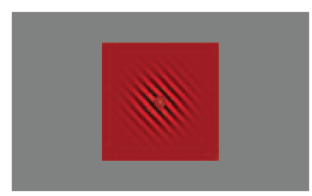

a) Orientation

b) Color



Color pattern

features

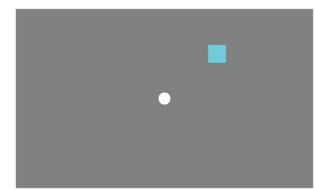

Faces, Bodies, Scenes, Flowers


a) Direction

b) Speed

a) Direction

b) None
a) Orientation
b) Spatial frequency
a) Orientation
b) Spatial frequency

a) $\mathrm{V} 1-\mathrm{V} 4, \mathrm{LO} 1$

b) $V_{1}, V_{2}, V_{3} A / B$

Parahippocampal a) $V_{1}$

$\begin{array}{ll}\text { b) Color } & \text { b) } \vee 1\end{array}$
Early visual
Posterior parietal

IPS2, IPS3

PCS

Early visual

a) Lateral occipital and temporal

Medial occipital

b) None

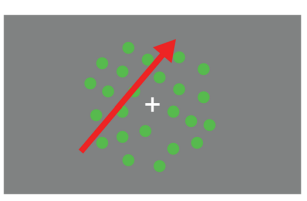

Direction

(cued by color)
Orientation

Direction
Superior frontal gyrus Supramarginal gyrus V1-V3

Intraoccipital sulcus

$\mathrm{MT+}$

V1, V2 
TABLE 1 | (Continued).

\begin{tabular}{llll}
\hline Reference & Stimuli & $\begin{array}{l}\text { Task-relevant } \\
\text { information }\end{array}$ & $\begin{array}{l}\text { Information } \\
\text { decoded } \\
\text { allowing decoding }\end{array}$
\end{tabular}

Ester et al. (2013)

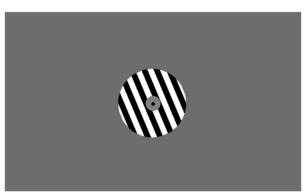

Orientation

Faces

Scenes

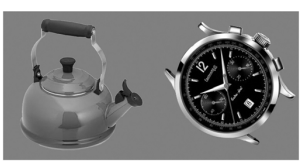

Exemplar identity

a) Visual features

b) Object name

Exemplar identity

Category

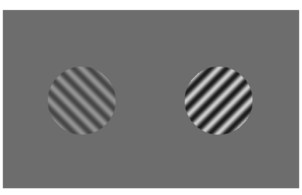

Stimulus contrast
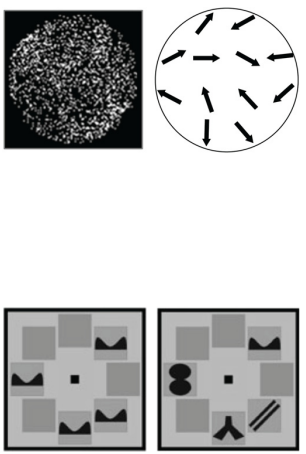

Exemplar identity with location
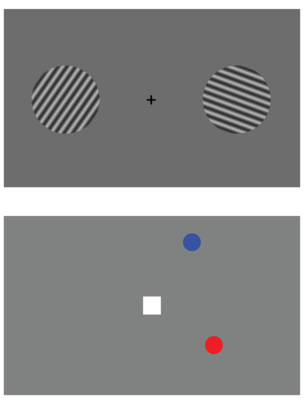

Faces

Scenes
Stimulus position

Position-specific orientation

Exemplar identity
a) Identity of whole object set b) Number of objects

Position-specific orientation

Stimulus position

Category
MT+

Posterior parietal Somatosensory

a) Right dorsolateral prefrontal

Premotor Left inferior frontal junction

Anterior cingulate Superior medial frontal Left sIPS, iIPS Left LOC

b) Left premotor sIPS, iIPS LOC

Contralateral V1, V2 Bilateral V3AB, V4

V1-V4, V3A

IPSO-IPS3

Superior PCS

Extrastriate visual cortex Lateral prefrontal cortex 
TABLE 1 | (Continued).

\begin{tabular}{|c|c|c|c|c|}
\hline Reference & Stimuli & $\begin{array}{l}\text { Task-relevant } \\
\text { information }\end{array}$ & $\begin{array}{l}\text { Information } \\
\text { decoded }\end{array}$ & $\begin{array}{l}\text { Cortical regions } \\
\text { allowing decoding }\end{array}$ \\
\hline Christophel et al. (2015) & & Color pattern features & Color pattern identity & $\begin{array}{l}\text { Early visual } \\
\text { Posterior parietal }\end{array}$ \\
\hline Ester et al. (2015) & + & Orientation & Orientation & $\begin{array}{l}\text { Bilateral V1, } \\
\text { Contralateral V4 } \\
\text { Ipsilateral IPS2, IPS3 } \\
\text { Prefrontal (incl. PCS) }\end{array}$ \\
\hline
\end{tabular}

Studies are organized first by date and then alphabetically by first author. Across studies, a wide range of visual stimuli have been employed, from oriented gratings to high-level stimuli such as faces, objects and scenes. We list both the task-relevant information as well as the information that could be decoded. In many cases, these are the same, but there are also some studies in which the level of decoding differed from the task-relevant information. For example, in several of the studies employing high-level visual stimuli, the task required maintenance of information about within-category exemplars (e.g., different faces or scenes), but the decoding was at the level of category (e.g., faces vs. scenes). In the final column, we list the major regions in which information could be decoded. Studies differed in how regions were identified (e.g., region-of-interest vs. searchlight analyses) and we adopt the level of description provided in the published studies. We ascribe decoding to particular functional regions (e.g., V1, MT, FFA) only if those regions were specifically localized. Further, note that we do not give any information about tested regions in which information could not be decoded. For this information, we refer readers back to the original cited papers. EBA, Extrastriate Body Area; FFA, Fusiform Face Area; IPSO-4, retinotopically-defined regions in and around the intra-parietal sulcus (ilPS, inferior intra-parietal sulcus; sIPS, superior intra-parietal sulcus); LOC, object-selective Lateral Occipital Complex; LO1, lateral occipital area 1; MT+, motion-selective areas including both the middle temporal (MT) and medial superior temporal (MST) areas; OFA, Occipital Face Area; PCS, precentral sulcus; PPA, Parahippocampal Place Area; RSC, scene-selective Retro Splenial Complex; TOS, scene-selective region near the Transverse Occipital Sulcus; V1-V4, retinotopically defined regions of early visual cortex.

In contrast to the ability to decode maintained information in visual cortex during working memory, studies investigating parietal and frontal cortex have often failed to find any evidence for maintained representations. For example, while Riggall and Postle (2012) could decode maintained information about motion direction in early visual cortex and MT+, this was not possible in frontal and parietal areas, even when selecting those areas that showed elevated activity during the delay. Similarly, Emrich et al. (2013) found that the ability to decode multiple items in memory decreased significantly with increasing load in early visual cortex and MT+, but could not decode remembered items in parietal cortex, even in those areas that showed loadsensitive delay period activity. These results argue strongly for the sensory recruitment model and suggest that neither elevated nor load-sensitive delay activity is a sufficient marker for maintained representations in working memory.

However, these failures to find evidence for maintained representations outside visual cortex should be treated cautiously since some studies have reported positive results (Christophel et al., 2012, 2015; Jerde et al., 2012; Lewis-Peacock and Postle, 2012; Han et al., 2013; Christophel and Haynes, 2014; Naughtin et al., 2014; Sprague et al., 2014; Ester et al., 2015). For example, in studies of working memory for colored patterns and motion flow patterns, Christophel and colleagues (Christophel et al., 2012, 2015; Christophel and Haynes, 2014), reported decoding of maintained information not only in early visual cortex but also in posterior parietal cortex. Further, decoding of stimulus position has been reported in both parietal and frontal cortex (Jerde et al., 2012; Sprague et al., 2014). While these results appear to disagree with the sensory recruitment model, they are potentially explained by considering the nature of the information that must be maintained and the underlying functional properties of the regions. Specifically, the novel stimuli employed by Christophel and colleagues are defined by the relative spatial position of the color or moving elements, precisely the kind of information that parietal cortex is generally thought to process during perception (Kravitz et al., 2011). Similarly, stimulus position is well represented in parietal and frontal cortex, related to sensory attention and motor behavior, making these regions a good substrate for maintaining representations of position in addition to early visual cortex. Taking into account that information may be maintained in brain regions more directly concerned with action, it has been suggested that "sensorimotor recruitment" rather than "sensory recruitment" may be a more appropriate way to think about maintained representations (D'Esposito and Postle, 2015).

Earlier we highlighted that the ability to maintain representations appears to be a general property of visual cortex. Given the evidence just discussed, it may be that this ability is not limited to visual cortex, but that any particular cortical region can be recruited for maintenance, depending on the nature of the information maintained. To test this idea, we presented participants sequentially with two visual objects before presenting a retro-cue (indicating which sample to hold in memory) and then asked them to perform one of two different tasks after a delay period (Lee et al., 2013). In the visual task participants were asked to indicate whether an object fragment presented after the delay belonged to the cued object or not, 
requiring the maintenance of visual features. In contrast, in the non-visual task, participants were asked to indicate whether a whole object presented after the delay was from the same subcategory or not, requiring the maintenance of the name or subcategory of the object. A separate behavioral experiment confirmed the nature of the information being maintained in the two tasks with visual object distractors presented in the delay period impairing performance on the visual-task more than the non-visual task and word distractors showing the opposite pattern. During the maintenance of visual properties, we found that object identity could be decoded from occipitotemporal but not prefrontal cortex. In contrast, during the maintenance of nonvisual properties (object category or name), we found that object identity could be decoded from prefrontal but not occipitotemporal cortex. These results confirm that information can be maintained in both prefrontal and visual cortex, but this maintenance is task-dependent and is stronger when the nature of the information matches the underlying functional properties of the region even for the same sample object. Further, the magnitude of activity in both regions was not modulated by task, providing further evidence that the magnitude of response during the delay period is dissociable from the presence or absence of maintained information.

One key prediction of the suggestion that information is maintained in regions that have functional properties matching the nature of that information is that there should be a correspondence between regions engaged during working memory and those engaged during perception of the same stimuli. For example, we suggested above that the decoding of maintained representations in posterior parietal cortex reported by Christophel et al. $(2012,2015)$ might reflect the complex visuospatial nature of their stimuli. We would therefore predict that those same regions should show strong decoding of the patterns during perception. Unfortunately, this was not tested in those studies. Similarly, it is unclear whether the parietal and frontal regions reported by Ester et al. (2015) also show decoding of orientation during perception.

More generally, it is possible that any region containing stimulus information during perception could maintain that information during working memory. In this context it is important to consider that, with sufficient power, stimulusrelated responses for a simple visual stimulation plus attention control task are observed in the vast majority of the brain (Gonzalez-Castillo et al., 2012). If information can be widely distributed during perception, then the same may be true of maintenance during working memory. The failure to find more distributed maintained representations could reflect lack of power. As is always the case, the current null results should be treated very cautiously. In our own work, showing taskdependent decoding during the delay in occipitotemporal and prefrontal cortex (Lee et al., 2013), the critical result is the relative strength of decoding, not the presence or absence of decoding in either task.

Overall, multivoxel decoding studies have provided strong support for the role of visual cortex in the maintenance of information during visual working memory. However, the ability to maintain representations is not just limited to visual cortex and may be a general property of cortex with the nature of the information maintained determining which regions are engaged. In some cases (e.g., position, orientation), the information may be well represented in multiple regions and the decoding of maintained content may be highly distributed. In other cases (e.g., faces, objects) the information may be maintained only in regions with more specialized functional properties. Critically, the ability to maintain information is dissociable from the presence or absence of delay activity and elevated activity may reflect separate functions related to attention, motor preparation or executive control.

\section{LIMITATIONS OF MULTIVOXEL DECODING}

Despite the advantages of decoding approaches for the study of maintenance during visual working memory, we need to be very cautious in interpreting the results (for discussion, see Serences and Saproo, 2012; Haynes, 2015).

First, although MVPA can provide evidence that there are distinct representations during visual working memory, it does not indicate what the nature of those representations are (Sligte et al., 2013). For example, Christophel and Haynes (2014) demonstrated decoding of maintained information about motion flowfields in $\mathrm{MT}+$, posterior parietal cortex and somatosensory cortex. It is unlikely that the underlying neural representations are similar in these three areas, but all three areas show distinct responses to the different flowfields that may reflect different aspects of the stimuli or associated cognitive processing.

Second, the success of MVPA depends on the spatial arrangement of responses across voxels and may require the presence of large-scale maps (Freeman et al., 2011). Thus in V1, properties such as position and orientation can be readily decoded. The failure to find decoding for particular information in a given region could simply reflect heterogeneous organization of that information across the cortex rather than its absence.

Reconstruction of stimuli based on an underlying encoding model (Serences and Saproo, 2012) has the advantage of an explicit model of the underlying neural responses, making the presence of decoding more interpretable. Further, since the model is fit at the individual voxel level, the method is not dependent on the large-scale organization of information. However, this approach is dependent on the specific a priori assumptions made in generating the model. The assumption of orientation tuning is very reasonable for early visual cortex, but it is much more challenging to generate a model for higher cognitive functions.

\section{RELATIONSHIP TO NON-HUMAN PRIMATE STUDIES}

In this section, we want to briefly discuss how the human multivoxel decoding results we have reviewed relate to findings in non-human primate literature, which have often focused on prefrontal cortex, and not visual cortex, as critical for the maintenance of information (for recent discussion, see also Postle, 2015b). 
First, while there is strong evidence from the fMRI studies we have reviewed for maintained representations in early visual cortex (e.g., Harrison and Tong, 2009; Serences et al., 2009) and MT+ (e.g., Riggall and Postle, 2012), there is only limited evidence for maintained signals in non-human primate V1 (Supèr et al., 2001) and MT (Bisley et al., 2004; Zaksas and Pasternak, 2006). One account could be that these varying results reflect the very different nature of the signals recorded-single unit spiking data from non-human primates vs. population threshold and sub-threshold neural activity reflected in the BOLD response. Consistent with this view, a recent study found that the amplitude of local field potential (LFP) oscillations in macaque MT do reflect the maintained motion direction (Mendoza-Halliday et al., 2014). However, it is worth noting that that same study did find evidence for maintained representations of motion direction in firing rate in MST in addition to lateral prefrontal cortex (Mendoza-Halliday et al., 2014).

Second, while non-human primate studies have often reported stimulus-selective sustained activity in prefrontal cortex (e.g., Funahashi et al., 1989; Freedman et al., 2003), some fMRI decoding studies have failed to find evidence for maintained representations in human prefrontal cortex (e.g., Riggall and Postle, 2012; Emrich et al., 2013). Our emphasis on the nature of the maintained information could explain some of the discrepancy since the "cat" vs. "dog" category task employed by Freedman et al. (2003) may require abstract category information similar to that required in our non-visual task, which emphasized object name or category and revealed decoding in prefrontal cortex (Lee et al., 2013). However, as in posterior areas, the different nature of the signals measured with fMRI and neurophysiological recordings may also help explain the apparent discrepancies. Recent work has started to emphasize the dynamics of firing rate changes in monkey prefrontal cortex (Stokes, 2015) and a population level re-analysis of the data collected by Freedman and colleagues (Meyers et al., 2008) revealed a complex relationship over time between information in single neurons and that in the population as a whole. Further, neurophysiological recordings have revealed that a broad range of differ types of task features are reflected in the responses of prefrontal neurons (Stokes et al., 2013; Lara and Wallis, 2014; Postle, 2015b) and it may be difficult to tease these apart in the population-level measures reflected in the fMRI BOLD signals.

Finally, another potential account of the apparent discrepancy between the human and monkey studies is highlighted by a recent study of monkeys with unilateral prefrontal lesions (Pasternak et al., 2015). These monkeys exhibited a contralesional deficit in maintaining motion information across a delay, which was substantially pronounced when rapid allocation of spatial attention was required. This deficit was delay specific, supporting a role of prefrontal cortex in maintenance. Combined with the direction-selective signals recorded in prefrontal cortex during the delay period (Zaksas and Pasternak, 2006), this result might suggest a role for prefrontal cortex in maintaining the motion information necessary for this task. However, the deficit in the lesioned monkeys was not dependent on the specific stimulus features (coherence of the sample stimulus), suggesting it did not involve sensory information. Instead given the pronounced impact of rapidly shifting attention, the authors suggest that the role of prefrontal cortex lies in attending and accessing the task-relevant motion signals that are maintained elsewhere. Thus, the single unit neurophysiology data from non-human primate prefrontal cortex may be more associated with attentional signals than stimulus properties, while the multivoxel decoding data in human posterior cortex primarily reflects maintained sensory representations. Support for a specific role of prefrontal cortex in representing attentional context has also been provided by at least one multi-voxel decoding study (Nelissen et al., 2013).

\section{RELATIONSHIP TO VISUAL MENTAL IMAGERY}

As we described earlier, the representation of information during visual working memory may be highly related to visual imagery. In both cases, visual information is represented in the absence of that information in the environment. The nature of the representations during visual imagery has been much debated (for review, see Pearson and Kosslyn, 2015). Recent evidence from multi-voxel decoding studies has provided strong support for the depictive (picture-like) view of visual imagery, which suggests visual imagery of a stimulus induces similar neural activation patterns with that generated by visual perception of the same stimulus (Stokes et al., 2009; Reddy et al., 2010; Cichy et al., 2012; Lee et al., 2012; Johnson and Johnson, 2014; Naselaris et al., 2015). For example, we trained participants to remember pictures of 10 common objects before placing them in the MRI scanner (Lee et al., 2012). During scanning, participants were cued with the name of the object and on interleaved trials were either presented with the picture of the object or asked to visually imagine the picture as vividly as possible. During imagery trials we found that we could decode the specific object the participant was imagining from responses in visual cortex. Furthermore, the patterns of response elicited during imagery were similar to those elicited during perception and it was possible to decode between imagery and perception suggesting that perception and imagery share similar substrates, much like the maintenance of information during visual working memory.

In comparing results from working memory with those from mental imagery it is worth noting that working memory paradigms involving a retro-cue, which requires the retrieval of previously presented information, are not that dissimilar from the paradigms used in mental imagery. The major difference is the time between presentation of the visual stimulation and the cue for retrieval.

To directly compare working memory and mental imagery, Albers et al. (2013) asked participants to perform two different tasks. In both cases, participants were first presented with a task cue followed by two serially presented gratings and then a second cue indicating which grating was relevant for that trial. In the working memory task, participants simply had to remember the cued grating over a delay period. 
Following the delay a probe stimulus was presented and participants indicated whether the probe was rotated clockwise or anticlockwise relative to the cued grating. In contrast, in the mental imagery task, participants had to mentally rotate the cued grating (with direction and angle indicated by the initial task cue) and then indicate whether the probe was rotated clockwise or anticlockwise relative to the imagined grating. Here the imagined grating is internally generated mental image that is novel but not remembered one. While Albers et al. (2013) refer to this as mental imagery, since the rotated image was never actually physically present, this task could also be interpreted as a short-term memory with manipulation task (i.e., requiring the working of "working memory"). They found that in V1-V3 they could decode orientation during the delay on both working memory and mental imagery trials. Furthermore, they could decode between tasks and there was also generalization to representations estimated during perception. These results suggest a common internal representation for visual working memory and mental imagery that is similar to that evoked during perception (Tong, 2013). Similar results were obtained by Christophel et al. (2015) with their color patterns, showing that transformed versions of the memorized stimulus could also be decoded from the same regions (early visual and posterior parietal cortex) as the original memorized stimulus.

In contrast to these results, Saad and Silvanto (2013) argued that working memory and visual imagery are partly dissociable processes. They asked participants to hold a grating in mind (visual short-term memory condition) or project it as a mental image on the computer screen (imagery condition), and compared the effect of each on visual perception. They found that both visual short-term memory (working memory) and imagery conditions were correlated with visual perception. However, while the subjective strength of visual imagery was negatively associated with visual perception, a positive correlation pattern was found for visual memory, suggesting dissociation. An alternative explanation for this is that the bottom-up visual input (screen), which is combined with the mental image (grating) in the imagery condition but not in the visual short-term memory condition, may interfere with visual stimuli for the visual perception performance. Thus, this dissociation may not reflect the different nature of signals for maintenance between

\section{REFERENCES}

Albers, A. M., Kok, P., Toni, I., Dijkerman, H. C., and de Lange, F. P. (2013). Shared representations for working memory and mental imagery in early visual cortex. Curr. Biol. 23, 1427-1431. doi: 10.1016/j.cub.2013. 05.065

Baddeley, A. (2012). Working memory: theories, models and controversies. Annu. Rev. Psychol. 63, 1-29. doi: 10.1146/annurev-psych-120710100422

Baddeley, A. D., and Hitch, G. (1974). "Working memory," in The Psychology of Learning and Motivation: Advances in Research and Theory, Vol. 8, ed. G. H. Bower (New York, NY: Academic Press), 47-89.

Bisley, J. W., Zaksas, D., Droll, J. A., and Pasternak, T. (2004). Activity of neurons in cortical area MT during a memory for motion task. J. Neurophysiol. 91, 286-300. doi: 10.1152/jn.00870.2003 imagery and working memory but interference effect between bottom-up visual inputs (Saad and Silvanto, 2013).

\section{CONCLUSION}

In this article, we have reviewed fMRI studies employing multivoxel decoding during working memory. These studies have revealed maintained stimulus representations during delays that are unrelated to elevated activity levels. While these studies have often highlighted the role of early visual cortex, this may in part reflect the simple stimuli commonly employed and not any privileged role of early visual cortex in the process of maintenance. We have highlighted studies reporting decoding of maintained information outside of visual cortex and suggest that the distribution of representations during visual working memory is dependent on the information maintained, reflecting both the stimulus and the task. Thus, even prefrontal cortex may exhibit maintained representations for some types of information. Further, we suggest there should be correspondence between regions containing information during perception and those containing information during working memory and that any region that contains information during perception may potentially contribute to maintained representations during working memory. While we have focused on the maintenance of information, it is important to remember that there are many other aspects of working memory task performance that regions may contribute to, including stimulus-response mappings, match-nonmatch status of a trial, motor programs and decision criteria. Importantly we suggest that there may not be a sharp divide between regions involved in maintenance and regions involved in representing these aspects of task performance, but that these functions can co-exist in the same regions.

\section{AUTHOR CONTRIBUTIONS}

S-HL and CIB planned, discussed and wrote this article together.

\section{FUNDING}

S-HL and CIB supported by the Intramural Research Program of NIMH (MH002909-08).

Brouwer, G. J., and Heeger, D. J. (2009). Decoding and reconstructing color from responses in human visual cortex. J. Neurosci. 29, 13992-14003. doi: 10. 1523/JNEUROSCI.3577-09.2009

Brouwer, G. J., and Heeger, D. J. (2011). Cross-orientation suppression in human visual cortex. J. Neurophysiol. 106, 2108-2119. doi: 10.1152/jn.00540.2011

Chafee, M. V., and Goldman-Rakic, P. S. (1998). Matching patterns of activity in primate prefrontal area $8 \mathrm{a}$ and parietal area 7ip neurons during a spatial working memory task. J. Neurophysiol. 79, 2919-2940.

Christophel, T. B., Cichy, R. M., Hebart, M. N., and Haynes, J.-D. (2015). Parietal and early visual cortices encode working memory content across mental transformations. Neuroimage 106, 198-206. doi: 10.1016/j.neuroimage.2014 11.018

Christophel, T. B., and Haynes, J. D. (2014). Decoding complex flow-field patterns in visual working memory. Neuroimage 91, 43-51. doi: 10.1016/j.neuroimage. 2014.01.025 
Christophel, T. B., Hebart, M. N., and Haynes, J.-D. (2012). Decoding the contents of visual short-term memory from human visual and parietal cortex. J. Neurosci. 32, 12983-12989. doi: 10.1523/jneurosci.0184-12.2012

Cichy, R. M., Heinzle, J., and Haynes, J. D. (2012). Imagery and perception share cortical representations of content and location. Cereb. Cortex 22, 372-380. doi: 10.1093/cercor/bhr106

Constantinidis, C., Franowicz, M. N., and Goldman-Rakic, P. S. (2001). The sensory nature of mnemonic representation in the primate prefrontal cortex. Nat. Neurosci. 4, 311-316. doi: 10.1038/85179

Courtney, S. M., Petit, L., Maisog, J. M., Ungerleider, L. G., and Haxby, J. V. (1998). An area specialized for spatial working memory in human frontal cortex. Science 279, 1347-1351. doi: 10.1126/science.279.5355.1347

Courtney, S. M., Ungerleider, L. G., Keil, K., and Haxby, J. V. (1997). Transient and sustained activity in a distributed neural system for human working memory. Nature 386, 608-611. doi: 10.1038/386608a0

Curtis, C. E., and D'Esposito, M. (2003). Persistent activity in the prefrontal cortex during working memory. Trends Cogn. Sci. 7, 415-423. doi: 10.1016/s13646613(03)00197-9

D'Esposito, M., and Postle, B. (2015). The cognitive neuroscience of working memory. Annu. Rev. Psychol. 66, 115-142. doi: 10.1146/annurev-psych010814-015031

Druzgal, T. J., and D’Esposito, M. (2003). Dissecting contributions of prefrontal cortex and fusiform face area to face working memory. J. Cogn. Neurosci. 15, 771-784. doi: 10.1162/089892903322370708

Dupont, P., Vogels, R., Vandenberghe, R., Rosier, A., Cornette, L., Bormans, G., et al. (1998). Regions in the human brain activated by simultaneous orientation discrimination: a study with positron emission tomography. Eur. J. Neurosci. 10, 3689-3699. doi: 10.1046/j.1460-9568.1998.00376.x

Emrich, S. M., Riggall, A. C., Larocque, J. J., and Postle, B. R. (2013). Distributed patterns of activity in sensory cortex reflect the precision of multiple items maintained in visual short-term memory. J. Neurosci. 33, 6516-6523. doi: 10. 1523/JNEUROSCI.5732-12.2013

Eriksson, J., Vogel, E. K., Lansner, A., Bergström, F., and Nyberg, L. (2015). Neurocognitive architecture of working memory. Neuron 88, 33-46. doi: 10. 1016/j.neuron.2015.09.020

Ester, E. F., Anderson, D. E., Serences, J. T., and Awh, E. (2013). A neural measure of precision in visual working memory. J. Cogn. Neurosci. 25, 754-761. doi: 10. 1162/jocn_a_00357

Ester, E. F., Serences, J. T., and Awh, E. (2009). Spatially global representations in human primary visual cortex during working memory maintenance. J. Neurosci. 29, 15258-15265. doi: 10.1523/JNEUROSCI.4388-09.2009

Ester, E. F., Sprague, T. C., and Serences, J. T. (2015). Parietal and frontal cortex encode stimulus- specific mnemonic representations during visual working memory parietal and frontal cortex encode stimulus-specific mnemonic representations during visual working memory. Neuron 87, 893-905. doi: 10 . 1016/j.neuron.2015.07.013

Freedman, D. J., Riesenhuber, M., Poggio, T., and Miller, E. K. (2003). A comparison of primate prefrontal cortex and inferior temporal cortices during visual categorization. J. Neurosci. 23, 5235-5246.

Freeman, J., Brouwer, G. J., Heeger, D. J., and Merriam, E. P. (2011). Orientation decoding depends on maps, not columns. J. Neurosci. 31, 4792-4804. doi: 10. 1523/JNEUROSCI.5160-10.2011

Funahashi, S., Bruce, C. J., and Goldman-Rakic, P. S. (1989). Mnemonic coding of visual space in the monkey's dorsolateral prefrontal cortex. J. Neurophysiol. 61, 331-349.

Funahashi, S., Bruce, C. J., and Goldman-Rakic, P. S. (1993). Dorsolateral prefrontal lesions and oculomotor delayed-response performance: evidence for mnemonic "scotomas". J. Neurosci. 13, 1479-1497.

Gonzalez-Castillo, J., Saad, Z. S., Handwerker, D. A., Inati, S. J., Brenowitz, N., and Bandettini, P. A. (2012). Whole-brain, time-locked activation with simple tasks revealed using massive averaging and model-free analysis. Proc. Natl. Acad. Sci. U S A 109, 5487-5492. doi: 10.1073/pnas.1121 049109

Greenlee, M. W., Magnussen, S., and Reinvang, I. (2000). Brain regions involved in spatial frequency discrimination: evidence from fMRI. Exp. Brain Res. 132, 399-403. doi: 10.1007/s002210000385

Han, X., Berg, A. C., Oh, H., Samaras, D., and Leung, H. C. (2013). Multivoxel pattern analysis of selective representation of visual working memory in ventral temporal and occipital regions. Neuroimage 73, 8-15. doi: 10.1016/j. neuroimage.2013.01.055

Harrison, A., Jolicoeur, P., and Marois, R. (2010). "What" and "where" in the intraparietal sulcus: an fMRI study of object identity and location in visual short-term memory. Cereb. Cortex 20, 2478-2485. doi: 10.1093/cercor/bhp314

Harrison, S. A., and Tong, F. (2009). Decoding reveals the contents of visual working memory in early visual areas. Nature 458, 632-635. doi: 10 1038 /nature 07832

Haynes, J.-D. (2015). A primer on pattern-based approaches to fMRI: principles, Pitfalls and perspectives. Neuron 87, 257-270. doi: 10.1016/j.neuron.2015. 05.025

Jerde, T. A., Merriam, E. P., Riggall, A. C., Hedges, J. H., and Curtis, C. E. (2012). Prioritized maps of space in human frontoparietal cortex. J. Neurosci. 32, 17382-17390. doi: 10.1523/JNEUROSCI.3810-12.2012

Jha, A. P., and McCarthy, G. (2000). The influence of memory load upon delayinterval activity in a working-memory task: an event-related functional MRI study. J. Cogn. Neurosci. 12, 90-105. doi: 10.1162/089892900564091

Johnson, M. R., and Johnson, M. K. (2014). Decoding individual natural scene representations during perception and imagery. Front. Hum. Neurosci. 8:59. doi: 10.3389/fnhum.2014.00059

Kravitz, D. J., Saleem, K. S., Baker, C. I., and Mishkin, M. (2011). A new neural framework for visuospatial processing. Nat. Rev. Neurosci. 12, 217-230. doi: 10. 1038/nrn3008

Lara, A. H., and Wallis, J. D. (2014). Executive control processes underlying multiitem working memory. Nat. Neurosci. 17, 876-883. doi: 10.1038/nn.3702

Lee, S.-H., Kravitz, D. J., and Baker, C. I. (2012). Disentangling visual imagery and perception of real-world objects. Neuroimage 59, 4064-4073. doi: 10.1016/j. neuroimage.2011.10.055

Lee, S.-H., Kravitz, D. J., and Baker, C. I. (2013). Goal-dependent dissociation of visual and prefrontal cortices during working memory. Nat. Neurosci. 16, 997-999. doi: 10.1038/nn.3452

Leung, H.-C., Gore, J. C., and Goldman-Rakic, P. S. (2002). Sustained mnemonic response in the human middle frontal gyrus during on-line storage of spatial memoranda. J. Cogn. Neurosci. 14, 659-671. doi: 10.1162/08989290260045882

Lewis-Peacock, J. A., and Postle, B. R. (2012). Decoding the internal focus of attention. Neuropsychologia 50, 470-478. doi: 10.1016/j.neuropsychologia. 2011.11.006

Linden, D. E. J., Bittner, R. A., Muckli, L., Waltz, J. A., Kriegeskorte, N., Goebel, R., et al. (2003). Cortical capacity constraints for visual working memory: dissociation of fMRI load effects in a fronto-parietal network. Neuroimage 20, 1518-1530. doi: 10.1016/j.neuroimage.2003.07.021

Linden, D. E. J., Oosterhof, N. N., Klein, C., and Downing, P. E. (2012). Mapping brain activation and information during category-specific visual working memory. J. Neurophysiol. 107, 628-639. doi: 10.1152/jn.00105.2011

Magnussen, S., and Greenlee, M. W. (1992). Retention and disruption of motion information in visual short-term memory. J. Exp. Psychol. Learn. Mem. Cogn. 18, 151-156. doi: 10.1037/0278-7393.18.1.151

Magnussen, S., and Greenlee, M. W. (1999). The psychophysics of perceptual memory. Psychol. Res. 62, 81-92. doi: 10.1007/s004260050043

Magnussen, S., Greenlee, M. W., Asplund, R., and Dyrnes, S. (1991). Stimulusspecific mechanisms of visual short-term memory. Vision Res. 31, 1213-1219. doi: 10.1016/0042-6989(91)90046-8

Marois, R. (2015). "The brain mechanisms of working memory: an evolving story," in Mechanisms of Sensory Working Memory: Attention and Performance XXV, eds P. Jolicoeur, C. Lefebvre, and J. Martinez-Trujillo (London: Academic Press), 23-31.

Martin, A. (2007). The representation of object concepts in the brain. Annu. Rev. Psychol. 58, 25-45. doi: 10.1146/annurev.psych.57.102904.190143

Martin, A. (2015). GRAPES-grounding representations in action, perception and emotion systems: how object properties and categories are represented in the human brain. Psychon. Bull. Rev. doi: 10.3758/s13423-015-0842-3 [Epub ahead of print].

Mendoza-Halliday, D., Torres, S., and Martinez-Trujillo, J. C. (2014). Sharp emergence of feature-selective sustained activity along the dorsal visual pathway. Nat. Neurosci. 17, 1255-1262. doi: 10.1038/nn.3785

Meyers, E. M., Freedman, D. J., Kreiman, G., Miller, E. K., and Poggio, T. (2008). Dynamic population coding of category information in inferior temporal and prefrontal cortex. J. Neurophysiol. 100, 1407-1419. doi: 10.1152/jn.90248.2008 
Miller, E. K., Erickson, C. A., and Desimone, R. (1996). Neural mechanisms of visual working memory in prefrontal cortex of the macaque. J. Neurosci. 16, 5154-5167.

Mitchell, D. J., and Cusack, R. (2008). Flexible, capacity-limited activity of posterior parietal cortex in perceptual as well as visual short-term memory tasks. Cereb. Cortex 18, 1788-1798. doi: 10.1093/cercor/bhm205

Naselaris, T., Olman, C. A., Stansbury, D. E., Ugurbil, K., and Gallant, J. L. (2015). A voxel-wise encoding model for early visual areas decodes mental images of remembered scenes. Neuroimage 105, 215-228. doi: 10.1016/j.neuroimage. 2014.10.018

Naughtin, C. K., Mattingley, J. B., and Dux, P. E. (2014). Distributed and overlapping neural substrates for object individuation and identification in visual short-term memory. Cereb. Cortex doi: 10.1093/cercor/bhu212 [Epub ahead of print].

Nelissen, N., Stokes, M., Nobre, A. C., and Rushworth, M. F. S. (2013). Frontal and parietal cortical interactions with distributed visual representations during selective attention and action selection. J. Neurosci. 33, 16443-16458. doi: 10. 1523/JNEUROSCI.2625-13.2013

Norman, K. A., Polyn, S. M., Detre, G. J., and Haxby, J. V. (2006). Beyond mind-reading: multi-voxel pattern analysis of fMRI data. Trends Cogn. Sci. 10, 424-430. doi: 10.1016/j.tics.2006.07.005

Oh, H., and Leung, H.-C. (2010). Specific and nonspecific neural activity during selective processing of visual representations in working memory. J. Cogn. Neurosci. 22, 292-306. doi: 10.1162/jocn.2009.21250

Pasternak, T., and Greenlee, M. W. (2005). Working memory in primate sensory systems. Nat. Rev. Neurosci. 6, 97-107. doi: 10.1038/ nrn1637

Pasternak, T., Lui, L. L., and Spinelli, P. M. (2015). Unilateral prefrontal lesions impair memory-guided comparisons of contralateral visual motion. J. Neurosci. 35, 7095-7105. doi: 10.1523/JNEUROSCI.526514.2015

Pearson, J., and Kosslyn, S. M. (2015). The heterogeneity of mental representation: ending the imagery debate. Proc. Natl. Acad. Sci. U S A 112, 10089-10092. doi: $10.1073 /$ pnas. 1504933112

Postle, B. R. (2015a). "Neural bases of the short-term retention of visual information," in Mechanisms of Sensory Working Memory: Attention and Performance XXV, eds P. Jolicoeur, C. Lefebvre, and J. Martinez-Trujillo (London: Academic Press), 43-58.

Postle, B. R. (2015b). The cognitive neuroscience of visual short-term memory. Curr. Opin. Behav. Sci. 1, 40-46. doi: 10.1016/j.cobeha.2014.08.004

Pratte, M. S., and Tong, F. (2014). Spatial specificity of working memory representations in the early visual cortex. J. Vis. 14:22, 1-12. doi: 10.1167/ 14.3.22

Ranganath, C., DeGutis, J., and D'Esposito, M. (2004). Category-specific modulation of inferior temporal activity during working memory encoding and maintenance. Brain Res. Cogn. Brain Res. 20, 37-45. doi: 10.1016/j.cogbrainres. 2003.11.017

Ranganath, C., and D'Esposito, M. (2005). Directing the mind's eye: prefrontal, inferior and medial temporal mechanisms for visual working memory. Curr. Opin. Neurobiol. 15, 175-182. doi: 10.1016/j.conb.2005.03.017

Reddy, L., Tsuchiya, N., and Serre, T. (2010). Reading the mind's eye: decoding category information during mental imagery. Neuroimage 50, 818-825. doi: 10. 1016/j.neuroimage.2009.11.084

Riggall, A. C., and Postle, B. R. (2012). The relationship between working memory storage and elevated activity as measured with functional magnetic resonance imaging. J. Neurosci. 32, 12990-12998. doi: 10.1523/jneurosci.1892-12.2012

Saad, E., and Silvanto, J. (2013). Contrast and strength of visual memory and imagery differentially affect visual perception. PLoS One 8:e84827. doi: 10. 1371/journal.pone.0084827

Serences, J. T., Ester, E. F., Vogel, E. K., and Awh, E. (2009). Stimulus-specific delay activity in human primary visual cortex. Psychol. Sci. 20, 207-214. doi: 10. $1111 /$ j.1467-9280.2009.02276.x

Serences, J. T., and Saproo, S. (2012). Computational advances towards linking BOLD and behavior. Neuropsychologia 50, 435-446. doi: 10.1016/j. neuropsychologia.2011.07.013
Sligte, I. G., van Moorselaar, D., and Vandenbroucke, A. R. E. (2013). Decoding the contents of visual working memory: evidence for process-based and content-based working memory areas? J. Neurosci. 33, 1293-1294. doi: 10. 1523/JNEUROSCI.4860-12.2013

Sneve, M. H., Alnæs, D., Endestad, T., Greenlee, M. W., and Magnussen, S. (2012). Visual short-term memory: activity supporting encoding and maintenance in retinotopic visual cortex. Neuroimage 63, 166-178. doi: 10.1016/j.neuroimage. 2012.06.053

Sprague, T. C., Ester, E. F., and Serences, J. T. (2014). Report reconstructions of information in visual spatial working memory degrade with memory load. Curr. Biol. 24, 2174-2180. doi: 10.1016/j.cub.2014.07.066

Sreenivasan, K. K., Curtis, C. E., and D'Esposito, M. (2014a). Revisiting the role of persistent neural activity during working memory. Trends Cogn. Sci. 18, 82-89. doi: 10.1016/j.tics.2013.12.001

Sreenivasan, K. K., Vytlacil, J., and D’Esposito, M. (2014b). Distributed and dynamic storage of working memory stimulus information in extrastriate cortex. J. Cogn. Neurosci. 26, 1141-1153. doi: 10.1162/jocn_a_ 00556

Stokes, M. G. (2015). "Activity-silent" working memory in prefrontal cortex: a dynamic coding framework. Trends Cogn. Sci. 19, 394-405. doi: 10.1016/j.tics. 2015.05.004

Stokes, M. G., Kusunoki, M., Sigala, N., Nili, H., Gaffan, D., and Duncan, J. (2013). Dynamic coding for cognitive control in prefrontal cortex. Neuron 78, 364-375. doi: 10.1016/j.neuron.2013.01.039

Stokes, M., Thompson, R., Cusack, R., and Duncan, J. (2009). Top-down activation of shape-specific population codes in visual cortex during mental imagery. J. Neurosci. 29, 1565-1572. doi: 10.1523/JNEUROSCI.4657-08.2009

Supèr, H., Spekreijse, H., and Lamme, V. A. (2001). A neural correlate of working memory in the monkey primary visual cortex. Science 293, 120-124. doi: 10. 1126/science. 1060496

Todd, J. J., and Marois, R. (2004). Capacity limit of visual short-term memory in human posterior parietal cortex. Nature 428, 751-754. doi: 10. 1038/nature02466

Todd, J. J., and Marois, R. (2005). Posterior parietal cortex activity predicts individual differences in visual short-term memory capacity. Cogn. Affect. Behav. Neurosci. 5, 144-155. doi: 10.3758/CABN.5.2.144

Tong, F. (2013). Imagery and visual working memory: one and the same? Trends Cogn. Sci. 17, 489-490. doi: 10.1016/j.tics.2013.08.005

Vogel, E. K., and Machizawa, M. G. (2004). Neural activity predicts individual differences in visual working memory capacity. Nature 428, 748-751. doi: 10. 1038/nature02447

Wager, T. D., and Smith, E. E. (2003). Neuroimaging studies of working memory: a meta-analysis. Cogn. Affect. Behav. Neurosci. 3, 255-274. doi: 10.3758/CABN. 3.4.255

Xing, Y., Ledgeway, T., McGraw, P. V., and Schluppeck, D. (2013). Decoding working memory of stimulus contrast in early visual cortex. J. Neurosci. 33, 10301-10311. doi: 10.1523/JNEUROSCI.3754-12.2013

$\mathrm{Xu}, \mathrm{Y}$., and Chun, M. M. (2006). Dissociable neural mechanisms supporting visual short-term memory for objects. Nature 440, 91-95. doi: 10.1038/nature04262

Zaksas, D., and Pasternak, T. (2006). Directional signals in the prefrontal cortex and in area MT during a working memory for visual motion task. J. Neurosci. 26, 11726-11742. doi: 10.1523/jneurosci.3420-06.2006

Zarahn, E., Aguirre, G., and D’Esposito, M. (1997). A trial-based experimental design for fMRI. Neuroimage 6, 122-138. doi: 10.1006/nimg.1997.0279

Conflict of Interest Statement: The authors declare that the research was conducted in the absence of any commercial or financial relationships that could be construed as a potential conflict of interest.

Copyright $\odot 2016$ Lee and Baker. This is an open-access article distributed under the terms of the Creative Commons Attribution License (CC BY). The use, distribution and reproduction in other forums is permitted, provided the original author(s) or licensor are credited and that the original publication in this journal is cited, in accordance with accepted academic practice. No use, distribution or reproduction is permitted which does not comply with these terms. 